\title{
THE FINITE INTERSECTION PROPERTY ON ANNIHILATOR RIGHT IDEALS
}

\author{
J. M. ZELMANOWITZ ${ }^{1}$
}

\begin{abstract}
In this article the finite intersection property on annihilator right ideals will be shown to be an adequate substitute for more stringent chain conditions on such ideals. One application of the investigation will produce a new characterization of orders in semisimple artinian rings, another will generate new classes of absolutely torsion-free rings.
\end{abstract}

Until otherwise indicated all rings are arbitrary associative rings not necessarily possessing an identity element. To simplify the statements of the results, we expand the usual definition of a prime ideal to include the ring itself. For any subset $A$ of a ring $R$ we set $r(A)=(0: A)=\{r \in R \mid A r=0\}$, the right annihilator of $A ;(0: x)$ being written for $(0:\{x\})$. More generally, for $A$ and $B$ subsets of $R,(B: A)$ will denote $\{r \in R \mid A r \subseteq B\}$. We let $l(A)$ denote the left annihilator of $A$.

A ring $R$ is said to have the finite intersection property on right annihilators provided that whenever $r(A)=0$ for a right ideal $A \subseteq R$ there exists $x_{1}, \ldots, x_{n} \in A$ with $\cap_{i=1}^{n}\left(0: x_{i}\right)=0$. It is readily apparent that a ring which satisfies the descending chain condition on right annihilators possesses this property; for choosing $x_{1}, \ldots, x_{n} \in A$ with $\bigcap_{i=1}^{n}\left(0: x_{i}\right)$ minimal among all such intersections forces $\bigcap_{i=1}^{n}\left(0: x_{i}\right)=0$. The converse is false however. For instance a commutative subdirectly irreducible (i.e., having a unique minimal ideal) nil ring which is not nilpotent has the finite intersection property; in fact it satisfies the stronger requirement that $\cap_{x \in A}(0: x)=0$ implies that $(0: x)=0$ for some $x \in A$. But such a ring cannot satisfy the descending chain condition on annihilators, else by well-known theorem [3, Theorem 1] it would be nilpotent. For a specific example of such a ring one may take any subdirectly irreducible homomorphic image of $\bigoplus_{0<\alpha<1} F x_{\alpha}$ where $F$ is a field and multiplication is defined by $x_{\alpha} x_{\beta}=x_{\alpha+\beta}$ if $\alpha+\beta<1$ and 0 otherwise (see Example 3 of [1]). This example also demonstrates that finite intersection properties on annihilators cannot force the nilpotence of nil rings.

A ring will be called nonsingular if its right singular ideal $Z(R)$ is zero, where $Z(R)=\{a \in R \mid(0: a)$ is an essential right ideal $\}$.

Proposition 1. Assume (1) $r(P)=0$ for every prime ideal $P$ of $R$; and

(2) $R$ has the finite intersection property on right annihilators.

Received by the editors December 18, 1974 and, in revised form, March 27, 1975.

AMS (MOS) subject classifications (1970). Primary 16A34; Secondary 16A12, 16A18, 16A46.

Key words and phrases. Finite intersection property on right annihilators, descending chain condition on right annihilators, right Goldie ring, absolutely torsion-free ring, hereditary kernel functor.

${ }^{1}$ This research was supported in part by NSF Grant GP 34098.

(c) American Mathematical Society 1976 
Then given any nonzero right ideal $I$ of $R$ there exist $x_{1}, \ldots, x_{n} \in I$ with $\cap_{i=1}^{n}\left(0: x_{i}\right)=0$.

Proof. It suffices to show that $r(I)=0$ for all nonzero right ideals $I$. If this is not the case, then use the finite intersection property together with Zorn's lemma to choose a right ideal $P$ maximal with respect to $r(P) \neq 0$. Now $r(P+R P)=r(P)$, so $P=P+R P$ by the maximality of $P$, and thus $P$ is an ideal of $R$. In fact $P$ is a prime ideal. For if $A$ and $B$ are ideals of $R$ properly containing $P$ then $r(A)=r(B)=0$. Hence $r(A B)=0$, and it follows that $P \varsubsetneqq A B$. Thus $P$ is a prime ideal with $r(P) \neq 0$, a contradiction which establishes the conclusion.

COROLlary. The rings satisfying the hypotheses of Proposition 1 are prime nonsingular rings.

PRoof. For in particular $r(I)=0$ for every nonzero right ideal $I$, whence $R$ is prime. Also if $Z(R) \neq 0$ then we have the contradiction that $\cap_{i=1}^{n}\left(0: x_{i}\right)$ $=0$ for some $x_{1}, \ldots, x_{n} \in Z(R)$.

We call a ring $R$ prime (semiprime) right Goldie if $R$ is prime (semiprime), nonsingular, and finite dimensional. As is well known, these properties characterize right orders in simple (semisimple) artinian rings [2].

THEOREM 2. Assume (1) $r(P)=0$ for every prime ideal $P$ of $R$;

(2) $R$ has the finite intersection property on right annihilators;

(3) $R$ has a uniform right ideal.

Then $R$ is prime right Goldie.

Proof. In view of the preceding corollary it remains only to prove that $R$ is finite dimensional. Let $I$ be a uniform (i.e., 1-dimensional) right ideal of $R$. By Proposition 1, there exist $x_{1}, \ldots, x_{n} \in I$ with $\cap_{i=1}^{n}\left(0: x_{i}\right)=0$. It follows that the homomorphism $\Phi: R \rightarrow I^{(n)}$ defined by $\phi(r)=\left(x_{1} r, \ldots, x_{n} r\right)$ is an isomorphism. Hence by [2, Theorem 1.1] $R$ is finite dimensional.

With but slight modification these results can be extended to semiprime rings. We will therefore not give complete proofs.

Proposition 3. Assume (1) $P \cap r(P)=0$ for every prime ideal $P$ of $R$;

(2) $R$ has the finite intersection property on right annihilators.

Then given any right ideal. I there exist $x_{1}, \ldots, x_{n} \in I$ with

$$
\bigcap_{i=1}^{n}\left(0: x_{i}\right) \cap I=0 .
$$

In particular, $R$ is a semiprime nonsingular ring.

PROof. If the conclusion fails then one can use Zorn's lemma to choose a right ideal $P$ maximal with respect to the property that $r(F) \cap P \neq 0$ for all finite subsets $F$ of $P$. If $G$ is a finite subset of $P+R P$, then there exists a finite subset $F$ of $P$ with $r(F) \subseteq r(G)$, and from this it follows that $P=P+$ $R P$, so $P$ is an ideal.

Next, from the finite intersection property one has that $r(P) \neq 0$. Furthermore $P \cap r(P) \neq 0$. Else $P \varsubsetneqq P+r(P)$, whence there exists a finite subset $H \subseteq P+r(P)$ such that $r(H) \cap(P+r(P))=0$. From $H$ one obtains finite subsets $F \subseteq P$ and $G \subseteq r(P)$ with $H \subseteq F+G$. Now 


$$
\begin{aligned}
H(r(F) \cap P) & \subseteq F(r(F) \cap P)+G(r(F) \cap P) \\
& =G(r(F) \cap P) \subseteq r(P) P \subseteq r(P) \cap P=0 .
\end{aligned}
$$

So $r(F) \cap P \subseteq r(H) \cap P=0$, contradicting the choice of $P$.

If $A$ and $B$ are ideals properly containing $P$, it follows that $r(A) \cap A=0$ $=r(B) \cap B$. Also $r(A B) \cap(A \cap B)=0$. For if $x \in r(A B) \cap(A \cap B)$, then $B x \subseteq r(A) \cap A=0$ whence $x \in r(B) \cap B=0$. Finally $P \varsubsetneqq A B$; for if $P=A B$ then $0 \neq r(P) \cap P \subseteq r(A B) \cap(A \cap B)=0$. So $P$ is a prime ideal with $P \cap r(P) \neq 0$, contradicting (1).

Additionally, we have $r(I) \cap I=0$ for every right ideal $I$, so $R$ is semiprime. Also if $Z(R) \neq 0$ there exist $z_{1}, \ldots, z_{n} \in Z(R)$ with $Z(R) \cap \cap_{i=1}^{n}\left(0: z_{i}\right)=0$, which is impossible since $\cap_{i=1}^{n}\left(0: z_{i}\right)$ is an essential right ideal. \|

As in Theorem 2 we get the following immediate consequence.

Theorem 4. Assume (1) $P \cap r(P)=0$ for every prime ideal $P$ of $R$;

(2) $R$ has the finite intersection property on right annihilators;

(3) $R$ has a faithful finite dimensional right ideal.

Then $R$ is semiprime right Goldie.

COROLlaRY. If $R$ is a prime (respectively, semiprime) ring satisfying the descending chain condition on right annihilators and possessing a uniform (respectively, faithful finite dimensional) right ideal, then $R$ is prime (respectively, semiprime) right Goldie.

Right absolutely torsion-free rings (abbreviated as ATF rings) were introduced in [4] as rings with identity elements in which for every kernel functor (synonym: left exact preradical) $\sigma$ on right $R$-modules with $\sigma(R)$ $\neq R, \sigma(R)=0$. See [4] or [5] for the terminology used here. It is straightforward to see that $R$ is a right ATF ring if and only if given any nonzero right ideal $I$ of $R, R$ can be embedded in $I^{(n)}=I \oplus \cdots \oplus I$ for some positive integer $n$ (equivalently, there exist $x_{1}, \ldots, x_{n} \in I$ with $\left.\cap_{i=1}^{n}\left(0: x_{i}\right)=0\right)$. See [5] for a proof of this and other characterizations. We remark that all of what follows is valid for rings without identity elements, provided appropriate modifications are made. However for the sake of simplicity we henceforth assume that rings contain identity elements.

In this terminology, Proposition 1 can be restated as follows.

PROPOSITION 5. If $R$ is a prime ring satisfying the finite intersection property on right annihilators then $R$ is right ATF.

COROllary. A prime ring which satisfies the ascending chain condition on left annihilators is right $A T F$.

Proof. The ascending chain condition on left annihilators is equivalent to the descending chain condition on right annihilators. \|

For convenience, let us call a right ideal $I$ exceptional if $R$ cannot be embedded in $I^{(n)}$ for any positive integer $n$. The next result follows directly from an examination of the proof of Proposition 1.

Proposition 6. $R$ is right ATF if and only if every nonzero prime ideal of $R$ is not exceptional. 
Proposition 7. If $P$ is a maximal exceptional right ideal of $R$, then $P$ is a prime ideal, either $r(P)=0$ or $P=\operatorname{lr}(P), R / P$ is right $A T F$, and $P=\sigma(R)$ where $\sigma$ is the kernel functor associated to the topologizing filter $\mathfrak{F}=\{I \mid I$ is a right ideal containing $\cap_{i=1}^{n}\left(0: p_{i}\right)$ for some $\left.p_{1}, \ldots, p_{n} \in P\right\}$.

Proof. This is trivial if $R$ is right ATF, so we may assume that $R$ is not ATF. As in the proof of Proposition 1, a maximal exceptional right ideal of $R$ is a prime ideal. If $P \neq \operatorname{lr}(P)$. then $\operatorname{lr}(P)$ is not exceptional, so there exists a monomorphism $f: R \rightarrow \operatorname{lr}(P)^{(n)}$ for some positive integer $n$. Since $f(r(P))$ $=f(1) r(P)=0$, it follows that $r(P)=0$.

To see that $R / P$ is right $\mathrm{ATF}$, let a right ideal $I \supsetneqq P$ be given. By the choice of $P$ there exists a finite subset $F \subseteq I$ with $r(F)=0$. Certainly $P \subseteq(P: F)$. If $P \neq(P: F)$, then there exists a finite set $G \subseteq(P: F)$ with $r(G)=0$. But $F G$ is a finite subset of $P$, whence $r(F G) \neq 0$, a contradiction. Thus $P=(P: F)$ which proves that $I / P$ is not exceptional in $R / P$. So $R / P$ is right ATF.

For the final statement of this proposition, recall that

$$
\sigma(R)=\{a \in R \mid(0: a) \in \mathfrak{F}\},
$$

from which it is obvious that $P \subseteq \sigma(R)$. Note that since $P$ is exceptional, $0 \notin \mathcal{F}$. If $P \neq \sigma(R)$ then there exist $x_{1}, \ldots, x_{n} \in \sigma(R)$ with $\cap_{i=1}^{n}\left(0: x_{i}\right)$ $=0$. So $0 \in \mathfrak{F}$, a contradiction which establishes the fact that $P=\sigma(R)$. $\|$

The author gratefully acknowledges the improvements suggested by the referee, and the assistance of L. W. Small in providing the example which appears at the beginning of this article.

\section{REFERENCES}

1. N. J. Divinsky, Rings and radicals, Mathematical Expositions, no. 14, Univ. of Toronto Press, Toronto, Ont., 1965. MR 33 \# 5654.

2. A. W. Goldie, Semi-prime rings with maximum condition, Proc. London Math. Soc. (3)10(1960), 201-220. MR 22 \#2627.

3. I. N. Herstein and L. W. Small, Nil rings satisfying certain chain conditions, Canad. J. Math. 16(1964), 771-776; addendum, ibid. 18(1966), 300-302. MR 29 \#3497; 32 \#690.

4. R. A. Rubin, Absolutely torsion-free rings, Bull. Amer. Math. Soc. 78(1972), 854-856. MR 45 \#8683.

5. J. Viola-Priolo, On absolutely torsion-free rings, Pacific J. Math. 56 (1975), 275-283.

Department of Mathematics, University of California, Santa Barbara, California 93106 